\title{
Exploring inside-out Doppler tomography: non-magnetic cataclysmic variables
}

\author{
E. J. Kotze ${ }^{1,2}$, S. B. Potter ${ }^{1}$, and V. A. McBride ${ }^{1,2}$ \\ 1 South African Astronomical Observatory, PO Box 9, Observatory 7935, 7925 Cape Town, South Africa \\ e-mail: ejk@saao.ac.za \\ 2 Astrophysics, Cosmology and Gravity Centre (ACGC), Department of Astronomy, University of Cape Town, Private Bag X3, \\ 7701 Rondebosch, South Africa
}

Received 22 April 2015 / Accepted 25 May 2015

\begin{abstract}
Context. Doppler tomography is a technique that has revolutionised the interpretation of the phase-resolved spectroscopic observations of interacting binary systems.

Aims. We present the results of our investigation of reversing the velocity axis to create an inside-out Doppler coordinate framework with the intent to expose overly compacted and enhance washed out emission details in the standard Doppler framework.

Methods. The inside-out tomogram is constructed independently of the standard tomogram by directly projecting phase-resolved spectra onto an inside-out velocity coordinate frame. For the inside-out framework, the zero-velocity origin is transposed to the outer circumference and the maximum velocities to the origin of the velocity space. We test the technique on a simulated system and two real systems with easily identifiable features, namely the accretion disc and bright spot in WZ Sge, and spiral shocks in IP Peg.

Results. Our tests show that there is a redistribution of the relative brightness of emission components throughout the tomograms, i.e., where the standard framework tends to concentrate and enhance lower velocity features towards the origin, the inside-out velocity framework tends to concentrate and enhance higher velocity features towards the origin. Conversely, the standard framework disperses and smears the higher velocities farther away from the origin whereas the inside-out framework disperses and smears the lower velocities. In addition, the projection of the accretion disc in velocity space now appears correctly orientated with the inner edge close to the maximum velocity origin and its outer edge closer to the zero-velocity outer circumference. Furthermore, the gas stream and secondary star are projected on the outside of the disc with the bright spot of the stream-disc impact region on the disc's outer edge in the inside-out velocity space.

Conclusions. We conclude that inside-out Doppler tomography complements the already powerful tomographic techniques in the analysis of spectroscopic emissions from interacting binary systems.
\end{abstract}

Key words. accretion, accretion disks - techniques: spectroscopic - binaries: close - novae, cataclysmic variables

\section{Introduction}

Cataclysmic variables (CVs) are interacting binary systems in which a white dwarf (primary) accretes material from a late-type main-sequence star (secondary). The secondary fills its Roche lobe and material flows through the inner Lagrangian point $\left(L_{1}\right)$ towards the primary. The material may form an accretion disc around the primary before it is finally accreted (see Warner 1995, for a comprehensive review of CVs).

Marsh \& Horne (1988) developed Doppler tomography as a technique aimed at constructing a two-dimensional velocity image (tomogram) of the accretion discs of CVs using an emission line in its spectra sampled at a number of orbital phases. Doppler tomography has revolutionised the interpretation of orbitally phase-resolved spectroscopic observations of interacting binary systems. It has become a valuable tool for resolving the distribution of line emission in CVs and other binary systems (see, e.g., Marsh 2001, Astrotomography workshop review). Significant contributions have been, amongst others, the discovery of two-arm spiral shocks in the accretion disc of the dwarf nova IP Peg (Steeghs et al. 1997) and mapping of the accretion stream in the polar HU Aqr detailing the transition from ballistic to magnetic flow (Schwope et al. 1997). Recent literature also abounds with examples where emission from the secondary and the bright spot of the stream-disc impact region in
CVs have been resolved in Doppler tomograms, such as CTCV J1300-3052 (Savoury et al. 2012) and V455 And (Bloemen et al. 2013).

We present a complementary technique for an inside-out velocity projection for Doppler tomography. This paper expands on the preliminary results presented in Kotze \& Potter (2015). In Sect. 2 we first review the Cartesian coordinate frame used in Doppler tomography and introduce a polar coordinate frame for the new inside-out velocity space. Next, in Sect. 3 we compare the standard and inside-out Doppler tomograms for a simulated disc-accreting system and two real CV systems, WZ Sge and IP Peg. Also in Sect. 3, we investigate how the inside-out framework redistributes relative contrast levels amongst the various emitting components of observations of a variety of CV features. Finally, in Sect. 4 we provide a summary and conclusions.

\section{Velocity space}

\subsection{Velocity in Cartesian coordinates}

We first review the Cartesian coordinate frame that co-rotates with the binary system, as described by Marsh \& Horne (1988). The top panel of Fig. 1 shows the Cartesian spatial coordinates (normalised by the binary separation $a$ ) for a model CV with an accretion disc. The assumed binary system parameters for 

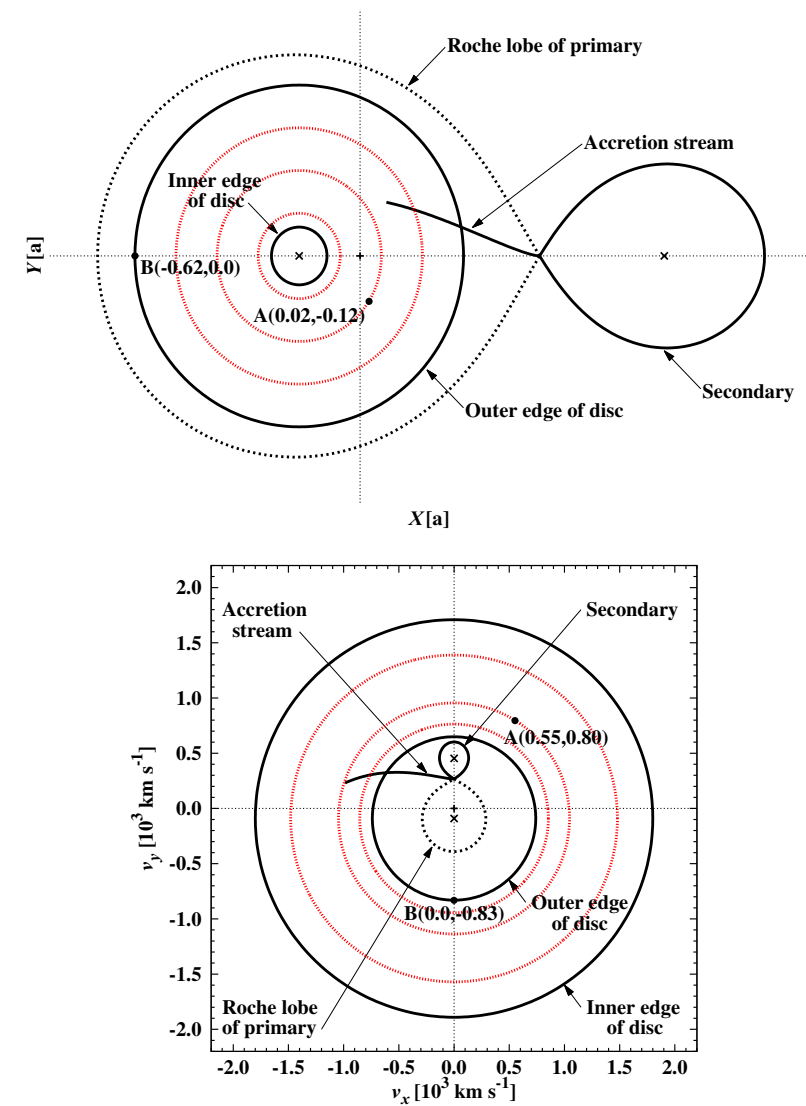

Fig. 1. Standard spatial and velocity Cartesian coordinates for a model CV. The C.O.M. of the binary is marked with a plus $(+)$ while that of the primary and secondary are marked with crosses $(\times)$. Overlays of the Roche lobe of the primary (dashed line), the accretion stream (solid line) from the secondary towards the primary (up to an azimuth angle of $30^{\circ}$ ), as well as radii at decrements of 0.25 times the outer disc radius are shown (red dashed circles). The outer disc radius is the 3:1 resonance radius while the inner disc radius is based on an absolute radial velocity of $1800 \mathrm{~km} \mathrm{~s}^{-1}$. There are also two arbitrary reference points, A and B, shown in the disc.

the model $\mathrm{CV}$ are: a primary mass of $0.85 M_{\odot}$, a mass ratio of $q=0.2$, an orbital period of $0.05669 \mathrm{~d}(82 \mathrm{~min})$ and an inclination of $77^{\circ}$. The origin is placed at the centre of mass (C.O.M.) of the binary (for a low $q$ system this is very close to the C.O.M. of the primary), the $X$-axis runs along a line through the centres of the primary and secondary, the $Y$-axis runs along a line parallel to the velocity vector of the secondary and the $Z$-axis along a line through the C.O.M. of the binary perpendicular to the orbital plane. In CVs where Roche-lobe overflow is considered to be responsible for the accretion stream and disc, these features are primarily confined to the orbital plane, therefore a two-dimensional layout in the orbital plane, i.e., the $X Y$-plane, is sufficient. The assumption that all motion is parallel to the orbital plane forms one of the postulates of Doppler tomography (Marsh 2001). The binary orbital phase zero is defined as the point of mid-eclipse of the primary by the secondary. The orbital motion is counter-clockwise around the C.O.M. of the binary.

Every spatial point $(x, y)$ in the system has a twodimensional Cartesian velocity coordinate $\left(v_{x}, v_{y}\right)$ as shown in the bottom panel of Fig. 1. Given the definition of the spatial frame it follows that, for example, the C.O.M. of the primary is moving in a negative $Y$-direction ( $v_{y}$ is negative), but with no movement in the $X$-direction ( $v_{x}$ is zero). Similarly, the

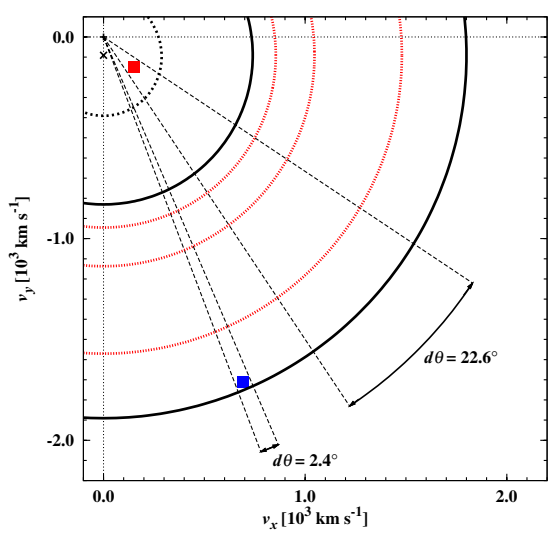

Fig. 2. Velocity bins in the Cartesian velocity framework (i.e., in the lower right quadrant of the Doppler map in Fig. 1). The figure shows the positions of a lower and a higher velocity pixel (red and blue, respectively).

C.O.M. of the secondary is moving in a positive $Y$-direction ( $v_{y}$ is positive), but also with no movement in the $X$-direction ( $v_{x}$ is zero). Structures such as the accretion stream and disc are more complex since they have a rotational velocity about the C.O.M. of the binary and a velocity due to structural motion. The increasing velocity of the accretion stream along its ballistic trajectory is clearly seen in the absolute increase in its $v_{x}$ component. Due to the Keplerian nature of the accretion disc its velocity profile appears reversed, i.e., the higher velocities of the inner edge of the disc are farther from the origin in velocity space and conversely for the lower velocities of the outer edge of the disc. The Keplerian nature of the disc is further emphasised by the radii at decrements of 0.25 times the outer disc radius. The secondary and ballistic stream also appear to be "inside" the disc in velocity coordinates.

We note that it is not possible to invert the velocity map to produce a spatial map, i.e., every velocity coordinate does not have a unique spatial coordinate making the inversion from velocity to spatial coordinates impossible without additional information (e.g., Harlaftis et al. 2004). In reality, orbitally phaseresolved spectroscopic observations are mapped directly onto the velocity map. Effectively each pixel, centred on a velocity coordinate $\left(v_{x}, v_{y}\right)$ in the velocity map, represents a velocity bin with a width $\left(\mathrm{d} v_{x}, \mathrm{~d} v_{y}\right)$. In the Cartesian coordinate frame of square pixels, $\mathrm{d} v_{x}=\mathrm{d} v_{y}$ for all velocity bins throughout the map. For example, in Fig. 2 we show a red and a blue pixel in the lower right quadrant of the Doppler map in Fig. 1 with velocities $\left(v_{x}, v_{y}\right)=(150,-150)$ and $(690,-1710) \mathrm{km} \mathrm{s}^{-1}$, respectively. Both pixels have the same velocity bin widths of $\mathrm{d} v_{x}=\mathrm{d} v_{y}=60 \mathrm{~km} \mathrm{~s}^{-1}$ corresponding to a range in velocity magnitude of $85 \mathrm{~km} \mathrm{~s}^{-1}$ between opposite velocity bin corners. However, the range in velocity angle $(\mathrm{d} \theta)$ is $22.6^{\circ}$ and $2.4^{\circ}$, respectively, i.e., the range in velocity angle decreases as a function of radial distance from the origin. Consequently, the lower radial velocity bins (pixels) sample a larger velocity vector range than the higher radial velocity bins (pixels).

The sinusoidal radial velocity curve $v_{\mathrm{r}}(\phi)$, traced by each velocity coordinate $\left(v_{x}, v_{y}\right)$, is a function of the orbital phase $\phi$ and centred on the mean (systemic) velocity $\gamma$ of the binary, which is given by:

$v_{\mathrm{r}}(\phi)=\gamma-v_{x} \cos 2 \pi \phi+v_{y} \sin 2 \pi \phi$.

To simplify the discussion this can be equally given in polar coordinates as:

$v_{r}(\phi)=\gamma-v \cos (\theta+2 \pi \phi)$, 

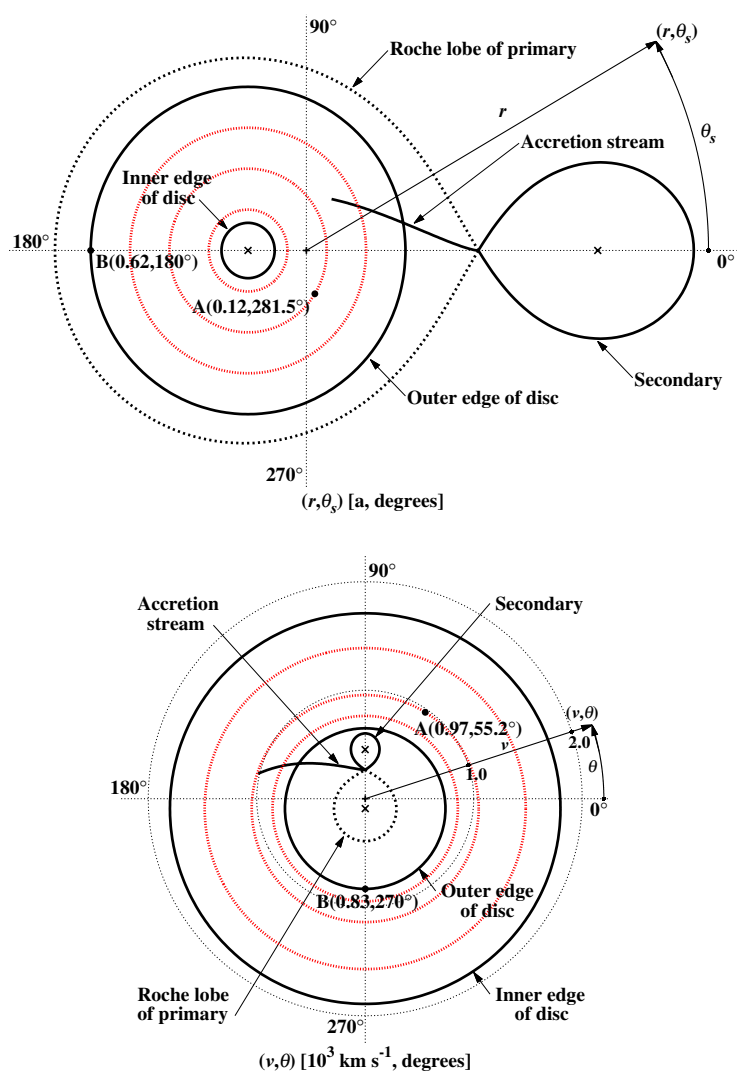

Fig. 3. As for Fig. 1 but in polar coordinates.

where $v$ is the velocity magnitude and $\theta$ is the velocity angle. The range in velocity magnitude of $85 \mathrm{~km} \mathrm{~s}^{-1}$ between opposite pixel (velocity bin) corners results in four different sinusoidal amplitudes. The range in amplitude is the same for both the red and blue pixels. However, the lower velocity red pixel subtends a larger velocity angle $(\theta)$ which corresponds to a larger range in the relative phases of the four sinusoids and therefore bound a larger area in the radial velocity-orbital phase space. Conversely, the blue pixel has a narrower bounding area in the radial velocity-orbital phase space. The implications of this effect is discussed in later sections.

\subsection{Velocity in polar coordinates}

We continue our discussion in the polar coordinate system as this is more conducive to the circularly symmetric nature of Doppler tomograms and gives a more direct indication of velocities and directions. The same CV model shown in Fig. 1 is shown in Fig. 3, but in spatial and velocity polar coordinates. Spatial coordinates (normalised by the binary separation $a$ ) are now given as the radial distance $r$ from the C.O.M. of the binary and the polar angle $\theta_{\mathrm{s}}$ (not to be confused with $\theta$ in the velocity frame) measured in an anti-clockwise direction from the line between the C.O.M. of the binary and the secondary (Fig. 3 top panel). The corresponding velocity map in polar coordinates is shown in the bottom panel of Fig. 3, again with the same model CV as for Fig. 1. Velocity magnitude $v$ increases as a linear function of distance from the origin with a direction given by the polar angle $\theta$ in an anti-clockwise direction measured from the line drawn from the C.O.M. of the binary horizontally to the right. All the $\mathrm{CV}$ model parameters and the arbitrary points A and $\mathrm{B}$ are the same as in the previous section. Therefore, for example, the secondary has a velocity magnitude of $400 \mathrm{~km} \mathrm{~s}^{-1}$
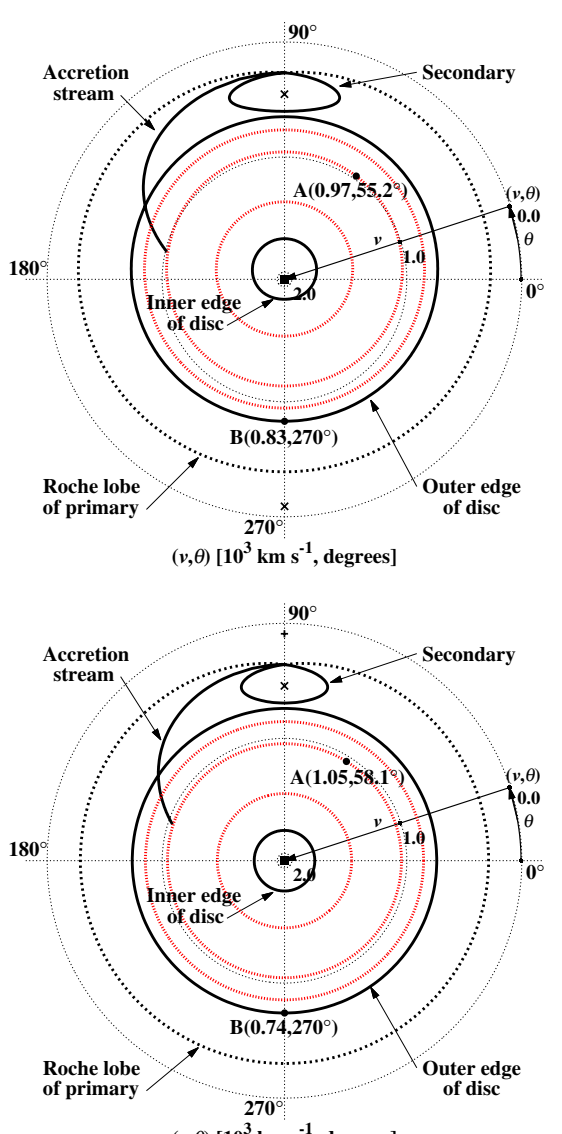

$(v, \theta)\left[10^{3} \mathrm{~km} \mathrm{~s}^{-1}\right.$, degrees $]$

Fig. 4. Inside-out velocity polar coordinates. The top and bottom panels show the model CV centred on the anti-C.O.M. of the binary and the anti-C.O.M. of the primary, respectively. See text for full description.

with a velocity direction (angle) of $\theta=90^{\circ}$ (i.e., $v_{x}=0$ and $v_{y}=400 \mathrm{~km} \mathrm{~s}^{-1}$ in Cartesian coordinates).

\subsection{Velocity in inside-out polar coordinates}

Having described the velocity polar coordinate frame in the previous section, we now investigate constructing an inside-out velocity polar coordinate frame with zero velocity on the outer circumference and the maximum velocities around the centre of the coordinate frame. The velocity magnitude $v$ now increases as a linear function of distance from the zero velocity outer circumference towards the origin with a direction given by the polar angle $\theta$. In order to make the inside-out framework complementary to the standard framework, and to facilitate a direct comparison between the frameworks, the default origin of the inside-out framework is set to the maximum radial velocity used to construct the standard framework. Effectively this is the maximum radial velocity extracted from the phase-resolved spectra of a source emission (or absorption) line, i.e., the "edge" of the data projected onto the framework to construct the tomogram.

The inside-out velocity map of the model CV presented in the previous figures is shown in the top panel of Fig. 4. Essentially the zero velocity origin (corresponding to the C.O.M. of the binary) is now transposed to the outer circumference and the maximum outer velocities to the central origin, giving the new inside-out velocity framework. The central origin, however, represents a discontinuity and the inner 4 velocity bins (pixels) around it are therefore ignored. We would like to point out that the spatial map (Fig. 3 top panel) remains unchanged. 


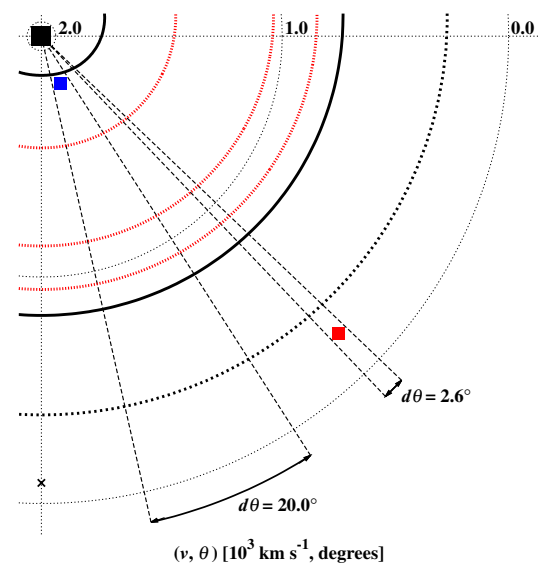

Fig. 5. Velocity bins in the inside-out polar velocity framework (i.e., in the lower right quadrant of the Doppler map in the top panel of Fig. 4). The figure shows the positions of a lower and a higher velocity pixel (red and blue, respectively). These pixels are the inside-out polar equivalent of the Cartesian pixels shown in Fig. 2.

As one can see (Fig. 4 top panel) the inner and outer edges of the accretion disc are now correctly orientated. Also the ballistic stream is now on the outside of the disc and "curves" inwards as it accelerates towards the disc and primary. The Roche lobe of the secondary is also now on the outside of the disc albeit upside down. It is upside down because it is orbiting as a solid body with the outside moving faster than the inside, as opposed to the Keplerian velocities of an accretion disc and the ballistic velocities of the accretion stream. The Roche lobe of the primary is now the outer bounded circular ring area, i.e., between zero velocity and the dashed line, that also contains the C.O.M. of the primary (indicated by $\mathrm{a} \times$ ).

The Keplerian velocity radial profile of the disc is still visibly apparent as can be seen by the equidistant disc radii contours (Fig. 4 top panel). We explored replacing the linear velocity axes with a Keplerian velocity $(\propto 1 / \sqrt{r})$ profile in an attempt to produce a more equidistant velocity contour profile. However, a correct calculation of the Keplerian profile would require knowledge of the primary mass of the actual system under investigation. This is rarely known and would introduce a new parameter that would no longer give a map that is simply a direct projection of the observations.

We note that the inner edge of the accretion disc is not centred on the origin (Fig. 4 top panel). This is because the disc is centred on the C.O.M. of the primary, whereas the origin represents the anti-C.O.M. of the binary, i.e., the C.O.M. of the binary is effectively the outer circumference of zero velocity with its anti-C.O.M. at the origin. Hence the Keplerian velocities of the disc will be offset by an amount corresponding to the orbital motion of the primary. This offset is also present in standard velocity maps but is more apparent in the inside-out framework. The bottom panel of Fig. 4 shows the same model but with the orbital motion of the primary subtracted from the model velocity profile. This effectively places the primary on the zero velocity outer circumference and its anti-C.O.M. at the origin. The effects of centring Doppler tomograms on the C.O.M. of the binary or of the primary are investigated in the next section.

As described in Sect. 2.1 each velocity bin (square pixel) has the same velocity magnitude range; however, the velocity vector range decreases as a function of radial distance from the central origin. To demonstrate this for the inside-out framework we show in Fig. 5 the equivalent red and blue pixels shown in Fig. 2. In the inside-out framework the higher velocity pixels are closer to the origin and therefore sample a larger velocity vector range than in the standard framework and conversely for the lower velocity pixels. This can be seen clearly by comparing Figs. 2 and 5. The implications of this effect are discussed in the following sections.

\section{Doppler tomography: standard and inside-out projections}

For real CVs, the orbital and structural velocities can be observed in the Doppler-shifted variations in the multi-component emission (and absorption) lines of their optical spectra. The orbitally phase-resolved spectroscopy of individual spectral lines can be projected onto a predefined velocity framework to produce a Doppler tomogram. The interpretation of such tomograms would then proceed by comparison with overlays of model velocity profiles of the likes described in the previous section (e.g., Marsh et al. 1994; Wolf et al. 1998). Traditionally, Doppler tomograms are produced by a projection onto a Cartesian coordinate frame, but given the circularly symmetric velocity profile of binary systems we elected to use a polar coordinate frame for our standard and inside-out tomograms as discussed in earlier sections.

We have modified the fast maximum entropy Doppler tomography code presented by Spruit (1998) to incorporate the inside-out velocity framework ${ }^{1}$. With the modified code the observed Doppler-shifted spectra are projected directly onto an inside-out velocity coordinate frame. All the standard and inside-out Doppler tomograms presented hereafter have been created using the unmodified and modified code, respectively.

\subsection{Simulated system: accretion disc, bright spot and secondary}

The results of applying standard and inside-out Doppler tomography to a simulated data set are shown in Fig. 6. A single line emission spectrum was constructed to simulate a disc-accreting system with an orbital period of $0.05669 \mathrm{~d}$ ( $82 \mathrm{~min})$, a mass ratio of $q=0.2$, a primary mass of $0.85 M_{\odot}$ and an inclination of $77^{\circ}$. The emission line was made of four components: two relatively narrow Gaussian profiles to represent emission from the accretion disc's bright spot and the irradiated face of the secondary star, a broad double peaked profile to represent emission from an accretion disc and some artificial noise. Appropriate phase-dependent Doppler shifts were then calculated for each of the components before summing to produce the trailed spectra shown in the top left panel of Fig. 6. The effect of inclination was also taken into account.

The binary system parameters listed above were used to calculate the model velocity profile overlay shown in all the tomograms. Included in the overlay are the Roche lobes of the primary (dashed line), the secondary (solid line) and a single particle ballistic trajectory (solid line) from the $L_{1}$ point up to $40^{\circ}$ in azimuth around the primary, as well as the 3:1 resonance radius as the outer edge of the accretion disc (large solid line circle). Also shown is an inner Keplerian radius (dot-dashed line) based on an absolute radial velocity of $1800 \mathrm{~km} \mathrm{~s}^{-1}$. This represents the maximum absolute radial velocity seen in the emission line variations.

\subsubsection{Standard Doppler tomogram}

The middle left panel of Fig. 6 shows the standard Doppler tomogram. The ring-like feature corresponds to emission from

1 Our code may be obtained upon request. 

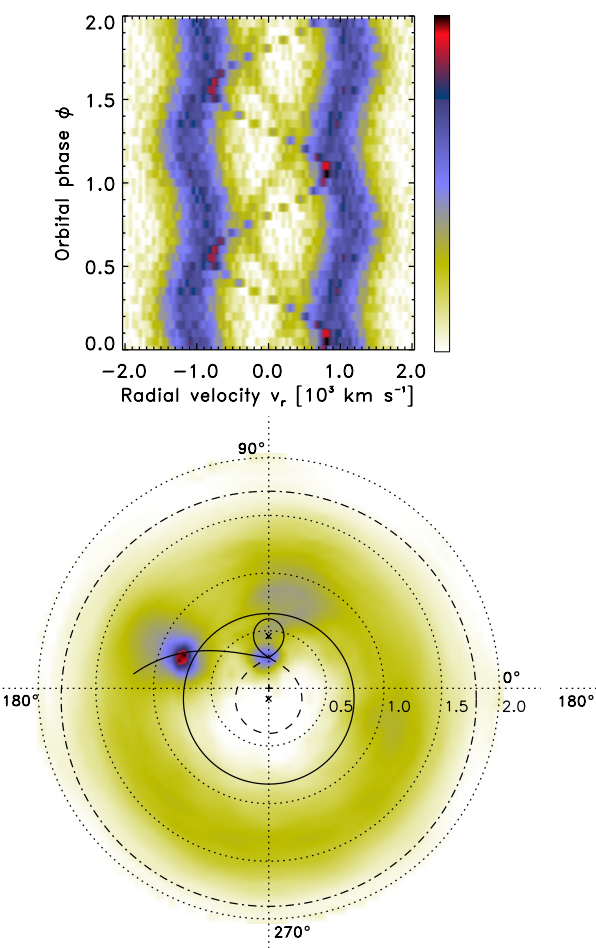

$(\mathrm{v}, \theta)\left[10^{3} \mathrm{~km} \mathrm{~s}^{-1}\right.$, degrees $]$

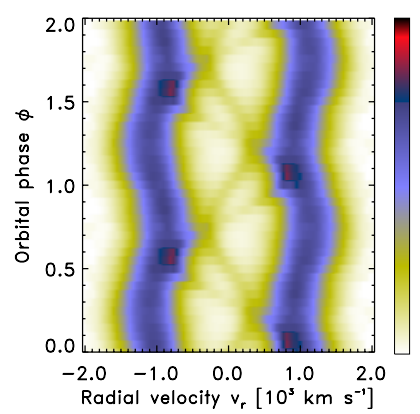

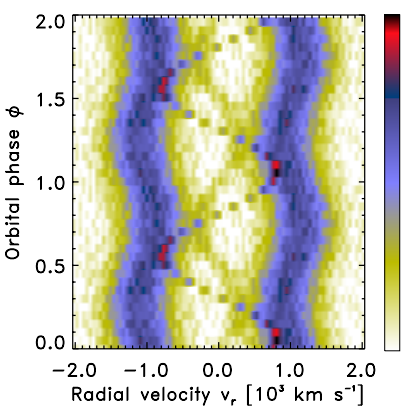

$90^{\circ}:$

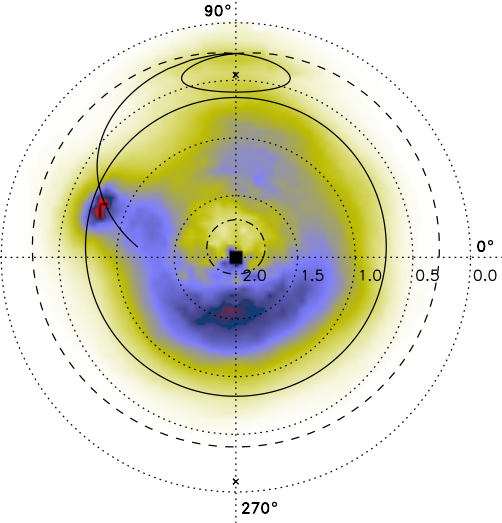

$(\mathrm{v}, \theta)\left[10^{3} \mathrm{~km} \mathrm{~s}^{-1}\right.$, degrees $]$

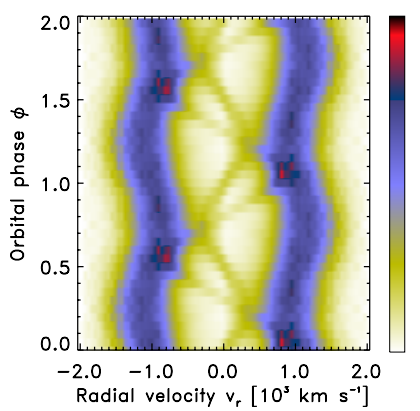

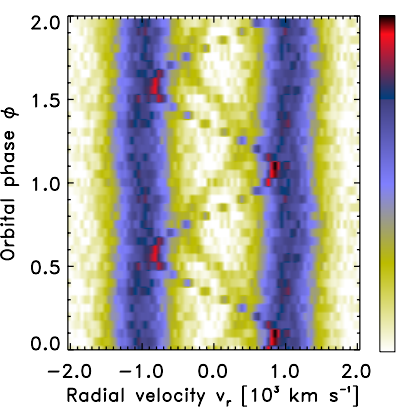

$90^{\circ}$

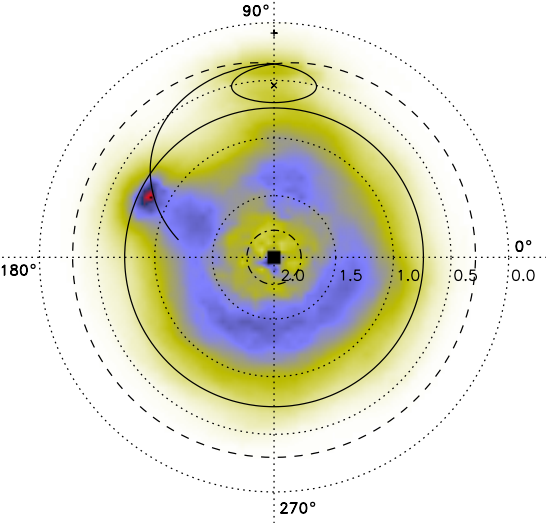

$(\mathrm{v}, \theta)\left[10^{3} \mathrm{~km} \mathrm{~s}^{-1}\right.$, degrees $]$

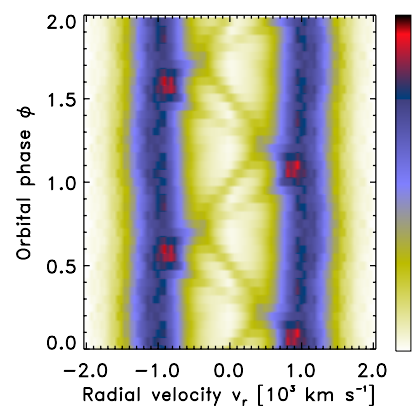

Fig. 6. Doppler tomography of synthetic spectra. The standard tomogram and two inside-out tomograms (with the anti-C.O.M. of the binary and of the primary at the origin, respectively) are shown from left to right in the middle panels for comparison (see text for full description). The top and bottom panels show the input and reconstructed trailed spectra, respectively, for the corresponding tomogram.

the disc with additional emission regions at the locations of the bright spot and secondary star. The lower left panel is the reconstructed trailed spectra from the Doppler code.

\subsubsection{Inside-out Doppler tomogram}

Anti-C.O.M. of binary at origin: the same trailed spectra (top middle panel) were used to produce the inside-out Doppler tomogram (middle panel). As expected from our modelling in previous sections, emission from the secondary star is now located on the outside of the disc and the bright spot on the disc's outer edge. Furthermore the disc has the "correct" orientation with a radial profile that has changed from a more even distribution in the standard tomogram, to a more inner disc concentration. This can be understood in terms of our discussion in Sect. 2, i.e., in the standard tomogram the higher velocity disc emission is distributed over more pixels covering a larger surface area. In the inside-out tomogram the higher velocity disc emission is concentrated into fewer pixels closer to the origin and hence appears brighter. This is perhaps more consistent with what one might expect from disc emission, i.e., the emitting volume in the inner edges are much smaller that the outer edges of the disc. However, the lower half of the disc appears to contain more emission at higher velocities than the upper half. This asymmetric artefact arises because the Keplerian motion of the accretion disc orbits the primary which in turn orbits the C.O.M. of the binary, i.e., the disc is projected off-centre. This asymmetry is also present in the standard tomograms but is enhanced in the inside-out tomograms due to the brightening of the high-velocity pixels around the origin.

Anti-C.O.M. of primary at origin: we next subtracted the orbital velocity of the primary $K_{1} \sim 91 \mathrm{~km} \mathrm{~s}^{-1}$ (e.g., Marsh et al. 1987) from the phase-resolved spectra (top right panel). As expected, the double peak emission from the disc has been "straightened" in the trailed spectra, i.e., with the orbital velocity of the primary removed, emission from a given radius in the disc shows a constant radial velocity in the trailed spectra. In the recalculated inside-out tomogram (middle right panel) the C.O.M. of the primary now effectively becomes the outer circumference of zero velocity with its anti-C.O.M. at the origin. Consequently, the disc is now centred on the origin and the asymmetry seen in the disc emission has disappeared as expected. 

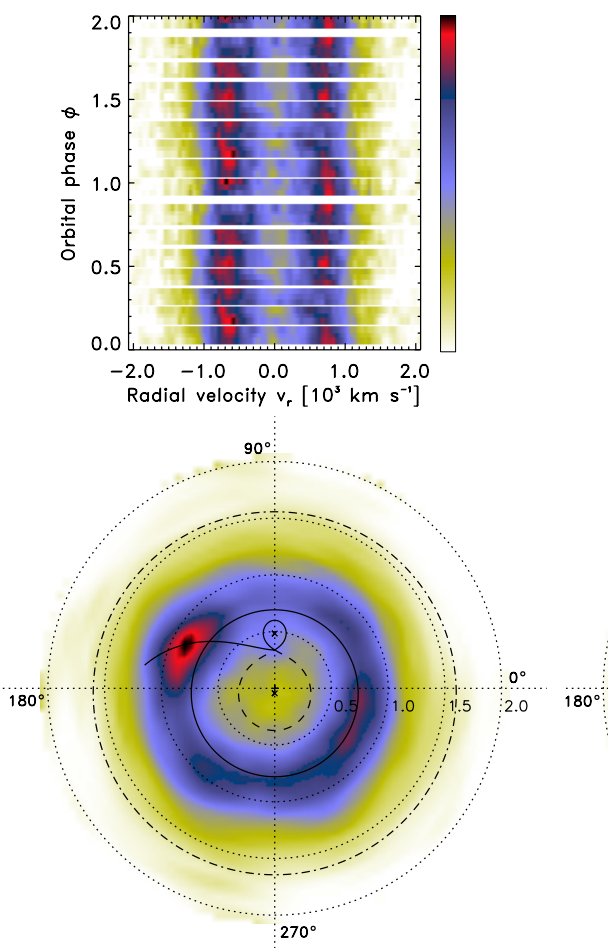

$(\mathrm{v}, \theta)\left[10^{3} \mathrm{~km} \mathrm{~s}^{-1}\right.$, degrees $]$

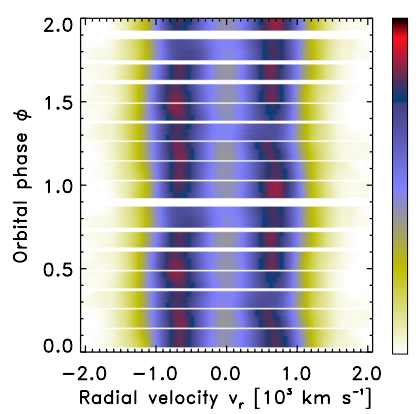

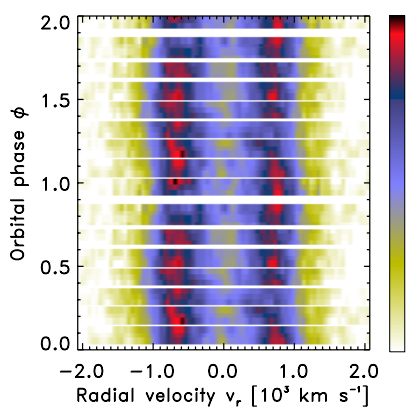

$90^{\circ}$

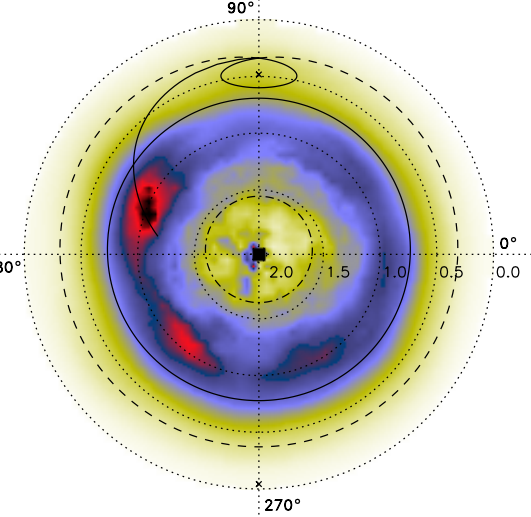

$(\mathrm{v}, \theta)\left[10^{3} \mathrm{~km} \mathrm{~s}^{-1}\right.$, degrees $]$

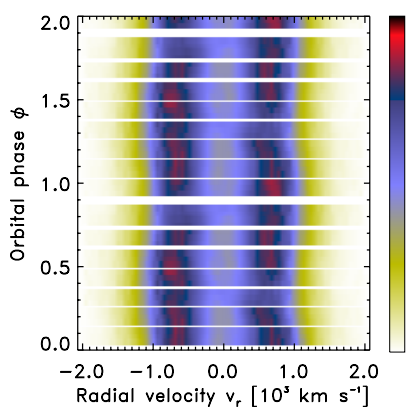

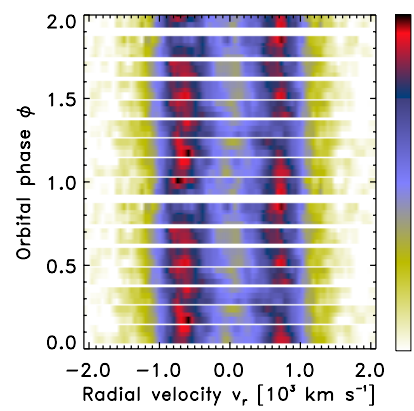

$90^{\circ}$

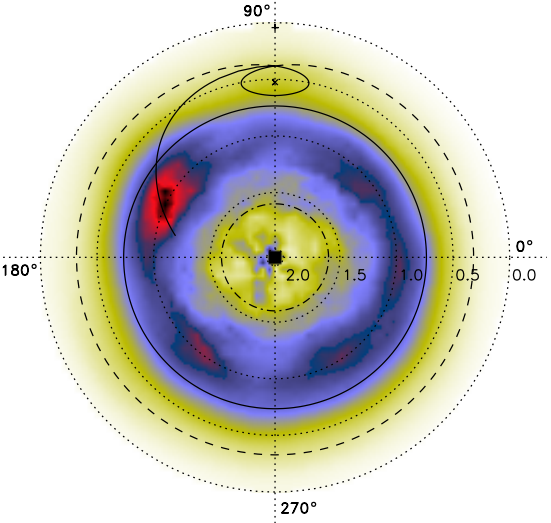

$(\mathrm{v}, \theta)\left[10^{\mathrm{s}} \mathrm{km} \mathrm{s}^{-1}\right.$, degrees $]$

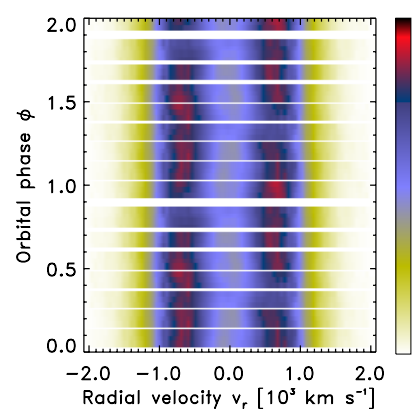

Fig. 7. Doppler tomography of WZ Sge in quiescence. Same layout as Fig. 6.

\subsection{WZ Sge in quiescence: accretion disc and bright spot}

WZ Sge is a prototypical member of the CV subclass referred to as WZ Sge-type dwarf novae (Bailey 1979). Doppler tomography of WZ Sge (e.g., Spruit \& Rutten 1998) has revealed a prominent accretion disc and bright spot where the ballistic accretion stream impacts the outer edge of the disc. This makes it an ideal test case for inside-out Doppler tomography.

The standard and inside-out Doppler tomography results for WZ Sge in quiescence are shown in Fig. 7 (similar layout to Fig. 6). The tomography is based on the $\mathrm{H} \alpha$ emission line from spectroscopic observations included with the fast maximum entropy Doppler tomography code (Spruit 1998). The model velocity profile overlay shown in all the tomograms was calculated using the orbital period of $0.056687846 \mathrm{~d}(82 \mathrm{~min})$ determined by Patterson et al. (1998), the inclination of $77^{\circ}$ obtained by Spruit \& Rutten (1998) and the mass parameters preferred by Steeghs et al. (2007), i.e., a primary mass of $0.85 M_{\odot}$ and a mass ratio $q=0.09$. The overlay includes the Roche lobes of the primary (dashed line), the secondary (solid line) and a single particle ballistic trajectory (solid line) from the $L_{1}$ point up to $45^{\circ}$ in azimuth around the primary, as well as the 3:1 resonance radius as the outer edge of the accretion disc (large solid line circle). An inner Keplerian radius (dot-dashed line) based on an absolute radial velocity of $1600 \mathrm{~km} \mathrm{~s}^{-1}$ which represents the maximum absolute radial velocity seen in the emission line variations, is also shown.

\subsubsection{Standard Doppler tomogram}

The most distinct features in the standard tomogram shown in the middle left panel of Fig. 7 are the relatively bright accretion disc and the bright spot of the impact region between the ballistic stream and disc. The disc appears brighter around the assumed outer edge (velocities lower than $1000 \mathrm{~km} \mathrm{~s}^{-1}$ ) while it becomes more diffused towards the assumed inner edge (velocities greater than $1000 \mathrm{~km} \mathrm{~s}^{-1}$ ). The extended nature of the bright spot (stream impact region) in velocity space is considered to be the result of the mixing of stream and disc material with different velocities (Marsh et al. 1990). The model stream lines up well with the brightest part of the impact region located at $\left(820 \mathrm{~km} \mathrm{~s}^{-1}, 150^{\circ}\right)$. There is only a slight enhancement in the emission at the expected velocity of the secondary $\left(400 \mathrm{~km} \mathrm{~s}^{-1}, 90^{\circ}\right)$.

\subsubsection{Inside-out Doppler tomogram}

Anti-C.O.M. of binary at origin: in the inside-out tomogram (middle panel) the disc is now correctly orientated with 
brighter disc emission occurring at generally higher velocities $\left(>1000 \mathrm{~km} \mathrm{~s}^{-1}\right)$. Due to the effect of the off-centre projection of the disc there is a slight enhancement in the brightness of the disc in the lower half. Although given WZ Sge's low mass ratio this effect is fairly minimal. Similar to the standard tomogram the bright spot (stream impact region) also dominates the brightness scale of the inside-out tomogram. However, it is now located towards the disc's outer edge in velocity space and has acquired an extended enhancement in the lower left quadrant covering $\left(1000 \mathrm{~km} \mathrm{~s}^{-1}, 180-250^{\circ}\right)$. The brightest part of the impact region it is now located at $\left(1050 \mathrm{~km} \mathrm{~s}^{-1}, 165^{\circ}\right)$, a slightly different velocity position than in the standard tomogram. This shift is caused by the redistribution of the relative contrast levels throughout the tomogram. The model stream, however, still lines up well with the brightest part of the impact region. The secondary is not discernible within the low-velocity emission covering $\left(0-700 \mathrm{~km} \mathrm{~s}^{-1}, 0-360^{\circ}\right)$. This is expected since it was barely discernible in the standard tomogram and is projected into more pixels in the inside-out tomogram. However, by subtracting the axisymmetric average of the emission at each radius around the origin (not shown), there is a patch of diffuse emission at the expected location of the secondary. Similar to the simulated system in the previous section, the high-velocity $\left(2000 \mathrm{~km} \mathrm{~s}^{-1}\right)$ noise present at the edge of the spectra is enhanced by the brightening of the high-velocity pixels around the origin.

Anti-C.O.M. of primary at origin: the velocity of the primary $K_{1} \sim 47 \mathrm{~km} \mathrm{~s}^{-1}$ with a phase zero offset 0.12 (Steeghs et al. 2007) was subtracted to obtain the phase-resolved spectra in the top right panel of Fig. 7. Given the relative low velocity of the primary that was subtracted, the "straightening" of the double peak emission from the disc in the trailed spectra is less pronounced compared to the case of the simulated system in the previous section. In the recalculated inside-out tomogram (middle right panel) the anti-C.O.M. of the primary is now at the origin. With the effect of the off-centre projection of the disc removed a more uniform circularly symmetric appearance of the disc is recovered. The extended enhancement of the bright spot in the lower left quadrant covering $\left(1000 \mathrm{~km} \mathrm{~s}^{-1}, 180-250^{\circ}\right)$ is also less pronounced.

\subsection{IP Peg in outburst: spiral shocks}

IP Peg is an eclipsing member of the dwarf nova subclass of CVs. Outbursts lasting 10-12 days occur every 70-100 days during which it brightens by 2-3 magnitudes. The spiral shocks observed in the accretion disc of IP Peg during an outburst (Steeghs et al. 1997) favour the disc instability model (Osaki 1974) as the cause of the recurrent outbursts. Since the disc expands during the outburst the outer disc will experience an increased gravitational attraction from the secondary. This causes perturbations in the circular Keplerian orbits of the disc material in the outer disc, leading to the formation of spiral arms in the disc. The spiral structure in the accretion disc of IP Peg makes it an ideal test case for inside-out Doppler tomography.

Standard and inside-out Doppler tomography results based on the He II ( $\lambda 4686 \AA$ ) emission line from the 1996 November spectroscopic observations taken during outburst maximum, are shown in Fig. 8 (similar layout to Figs. 7 and 6). These observations were presented first by Harlaftis et al. (1999) and the reader is referred to that paper for a detailed description of the data set and reduction procedures. All the tomograms in Fig. 8 show only the non-axisymmetric emission. The binary system parameters as calculated by Copperwheat et al. (2010), i.e., an orbital period of $0.1582061029 \mathrm{~d}(228 \mathrm{~min})$, an inclination of $83.8^{\circ}$, a primary mass of $1.16 M_{\odot}$ and a mass ratio $q=0.48$ were used to calculate the model velocity profile overlay shown in all the tomograms. Included in the overlay are the Roche lobes of the primary (dashed line), the secondary (solid line) and a single particle ballistic trajectory (solid line) from the $L_{1}$ point up to $45^{\circ}$ in azimuth around the primary, as well as the tidal radius as the outer edge of the accretion disc (large solid line circle). Also indicated is an inner Keplerian radius (dot-dashed line) based on an absolute radial velocity of $1200 \mathrm{~km} \mathrm{~s}^{-1}$, representing the maximum absolute radial velocity seen in the emission line variations.

\subsubsection{Standard Doppler tomogram}

In the standard tomogram shown in the middle left panel of Fig. 8 the emission from the leading side of the irradiated secondary is seen as a bright spot at $\left(200 \mathrm{~km} \mathrm{~s}^{-1}, 95^{\circ}\right)$ which appears to be "inside" the disc. There is also a low-velocity component visible at $\left(0-300 \mathrm{~km} \mathrm{~s}^{-1}, 140-250^{\circ}\right)$. This low-velocity component has been seen in other dwarf novae during outburst, but its origin is not understood (Steeghs et al. 1996). The origin of the high-velocity component seen as a diffuse patch of emission at $\left(1000-1500 \mathrm{~km} \mathrm{~s}^{-1}, 0^{\circ}\right)$ is also uncertain. The tomogram is dominated by the emission from the shocks in the accretion disc that creates an extended two-armed spiral structure. The two spiral shocks have a clear asymmetry in both brightness and velocity. The observed velocity asymmetry of the material in the shocks suggests a highly non-Keplerian flow in the disc since circular rings of emission are expected from a purely Keplerian disc. The shocks appear to be spiralling "outwards" from lower to higher velocities. The upper spiral shock extends to velocities lower than our velocity profile overlay of the outer disc radius. This supports the suggestion by Steeghs et al. (1997) that the disc possibly expanded beyond its tidal radius early on in the outburst as a result of it being triggered by a disc instability.

\subsubsection{Inside-out Doppler tomogram}

Anti-C.O.M. of binary at origin: IP Peg's mass ratio (0.48) is fairly high. Consequently the C.O.M. of the primary is significantly offset from the C.O.M. of the binary system. This can be clearly seen in the offset of the model overlay w.r.t. the origin in the inside-out tomogram (middle panel). As expected the secondary is seen as an extended diffuse patch of emission "outside" the disc. However, the low-velocity component visible at $\left(0-300 \mathrm{~km} \mathrm{~s}^{-1}, 140-250^{\circ}\right)$ in the standard tomogram is no longer discernible. The high-velocity patch of emission at (1000-1500 $\left.\mathrm{km} \mathrm{s}^{-1}, 0^{\circ}\right)$ has become more enhanced and compact given its new projection closer to the origin. The two-armed spiral structure associated with the shocks in the disc is still clearly present in the inside-out tomogram. However, the asymmetry in the brightness of the shocks caused by the off-centre projection of the disc is more pronounced than in the standard tomogram. The upper shock is more extended and diffuse while the lower shock is more compact and brighter.

Anti-C.O.M. of primary at origin: the phase-resolved spectra in the top right panel of Fig. 8 were obtained by subtracting the orbital velocity of the primary $K_{1} \sim 152 \mathrm{~km} \mathrm{~s}^{-1}$ (average from Copperwheat et al. 2010). Given the complex structure of the trailed spectra (Harlaftis et al. 1999) the "straightening" of the double peak emission from the disc is primarily seen in the blue peak. These "straightened" spectra were used as 

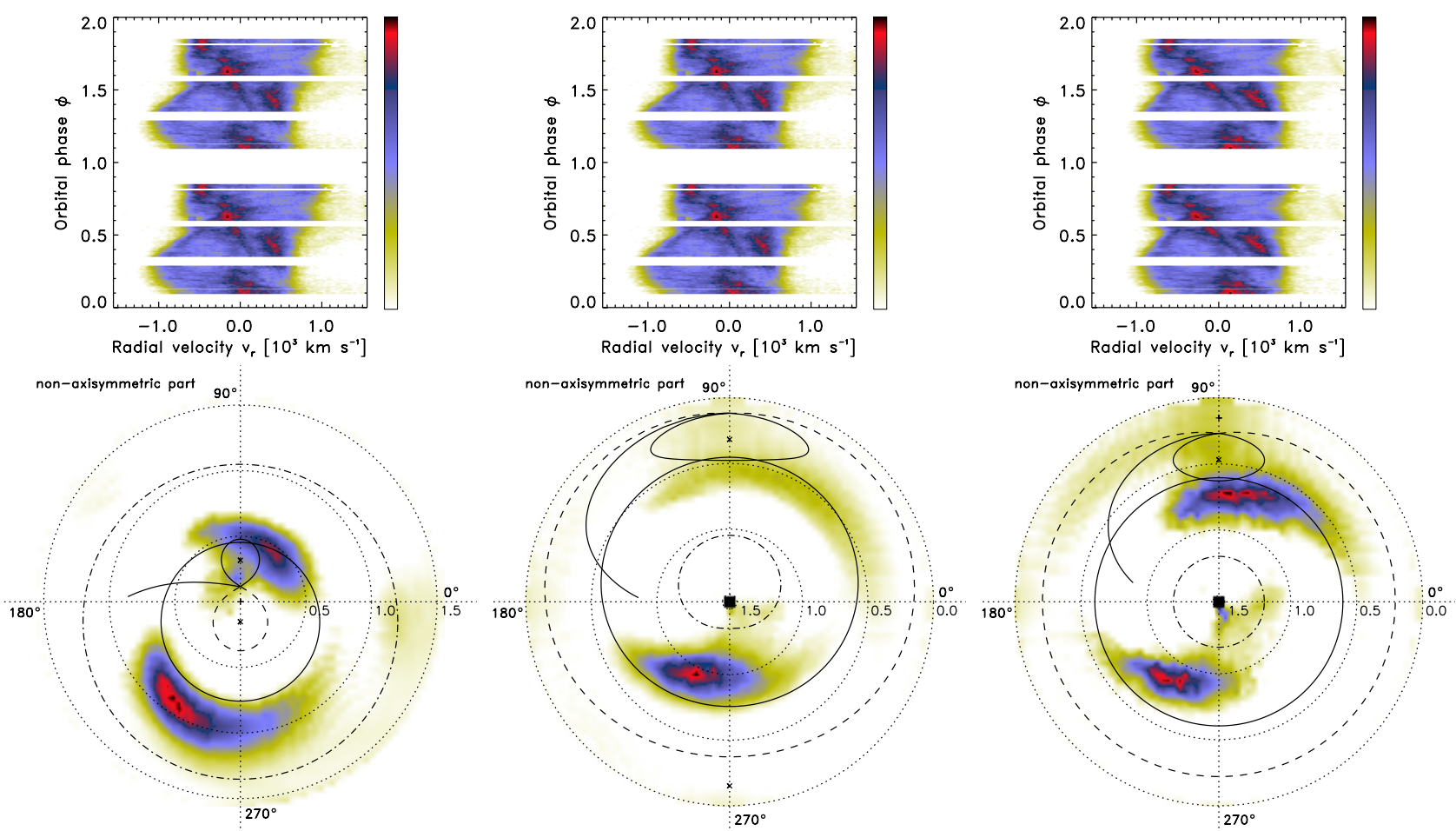

$(\mathrm{v}, \theta)\left[10^{3} \mathrm{~km} \mathrm{~s}^{-1}\right.$, degrees $]$

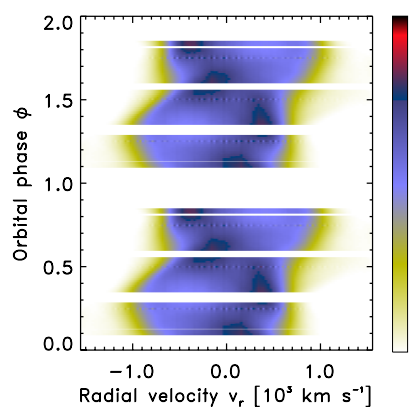

$(\mathrm{v}, \theta)\left[10^{\mathrm{s}} \mathrm{km} \mathrm{s}^{-1}\right.$, degrees $]$

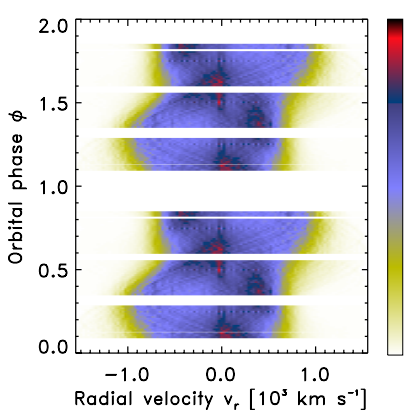

$(\mathrm{v}, \theta)\left[10^{3} \mathrm{~km} \mathrm{~s}^{-1}\right.$, degrees $]$

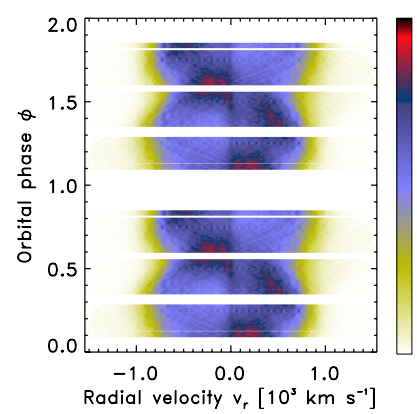

Fig. 8. Doppler tomography of IP Peg in outburst. Same layout as Figs. 7 and 6. Only the non-axisymmetric emission is shown in the tomograms.

input to produce the tomogram in the middle right panel. The model overlay clearly displays the removal of the C.O.M. offset. The secondary has become more compact and brighter. This is because the position of the secondary has moved significantly closer to the origin leading to its enhancement. Similarly the patch of high-velocity emission at $\left(1000-1500 \mathrm{~km} \mathrm{~s}^{-1}, 0^{\circ}\right)$ has become slightly more enhanced. Although the origin of this feature is unknown it is associated with the low brightness emission seen in the input trailed spectra at high radial velocity $\left(>1000 \mathrm{~km} \mathrm{~s}^{-1}\right)$ between phases 0.4 and 0.8 . In addition an extended low-velocity component is now visible at $\left(0-250 \mathrm{~km} \mathrm{~s}^{-1}, 110-150^{\circ}\right)$. This low-velocity component is part of the low-velocity component of unknown origin (Steeghs et al. 1996) covering $\left(0-300 \mathrm{~km} \mathrm{~s}^{-1}, 140-250^{\circ}\right)$ in the standard tomogram. The asymmetry between the brightness of the two shocks caused by the off-centre projection of the disc has also been removed. The spiral nature of the shocks is clearly evident and is more intuitive to interpret in respect that it shows how they begin at the outer edges of the disc and curve inwards towards the primary as they increase in velocity.

\section{Summary}

We have investigated the use of an inside-out velocity coordinate frame for Doppler tomograms of non-magnetic cataclysmic variables. In the inside-out framework zero velocity is on the outer circumference and the maximum velocities are around the centre of the coordinate frame. We modified the fast maximum entropy Doppler tomography code presented by Spruit (1998) to incorporate the inside-out framework which allowed us to construct inside-out tomograms independently of standard tomograms by directly projecting the observed spectra onto the inside-out velocity coordinate frame.

We applied our new inside-out velocity projection to real data of the dwarf nova system WZ Sge during a normal faint state. The accretion disc has the correct orientation with the bright spot located towards the disc's outer edge (in velocity space). In addition there is a redistribution of disc brightness, i.e., in the standard tomogram the disc appears to brighten for velocities lower than $1000 \mathrm{~km} \mathrm{~s}^{-1}$ whereas with the insideout velocity projection the disc brightens for velocities higher than $1000 \mathrm{~km} \mathrm{~s}^{-1}$. The difference arises because the line emission from the higher velocities emanating from the inner regions of the disc are spread out over a larger area with more pixels in the standard tomogram, whereas the higher velocity emission is concentrated in a smaller area with fewer pixels in the insideout tomogram as is more in line with a real disc brightness profile. Given the relative low mass ratio $(q \sim 0.1)$ of WZ Sge this real data set only marginally highlighted the vertical asymmetry in the brightness distribution in tomograms with an off-centre 
projection of the accretion disc, i.e., with the C.O.M. or antiC.O.M. of the binary at the origin. The asymmetry is somewhat enhanced in the inside-out velocity framework (anti-C.O.M. of the binary at the origin), but it can be removed by subtracting the orbitally induced Doppler velocity of the primary from the phase-resolved input spectra.

We also applied our new projection technique to real data of the dwarf nova system IP Peg during an outburst which shows emission from the irradiated face of the secondary and a distinct spiral structure in the accretion disc. The secondary is now placed "outside" the correctly orientated disc in velocity space. The dominant spiral structure of the shocks in the disc is well preserved and the shocks are now spiralling "inwards" from lower to higher velocities. Due to the relative high mass ratio $(q \sim 0.5)$ of IP Peg the vertical asymmetry in the brightness distribution in the tomograms with the C.O.M. or anti-C.O.M. of the binary at the origin is more pronounced than in the case of WZ Sge. This implies that it is crucial to consider the mass ratio $(q)$ of the system when interpreting the brightness distribution in a tomogram. The asymmetry in the brightness distribution is removed in tomograms with the anti-C.O.M. of the primary at the origin resulting in a more circularly symmetric brightness distribution. This, together with the "inwards" spiralling appearance of the shocks, creates a velocity image which is perhaps more intuitive with the expected radial disc profile of the shocks.

We conclude that our new technique of inside-out Doppler tomography is complementary to the existing technique. The standard projection used in Doppler tomography tends to concentrate and enhance lower velocity features while higher velocity features are more separated and dispersed. Conversely, the inside-out velocity projection tends to concentrate and enhance higher velocity features while lower velocity features are more separated and dispersed. This is perhaps more consistent with the emission distribution in disc-accreting $\mathrm{CVs}$ where the high- and low-velocity emissions are produced in smaller and larger fractions of the total system volume, respectively.

Acknowledgements. We thank Danny Steeghs for providing the IP Peg data and we are grateful to Keith Horne for his helpful comments. Additionally we thank the referee for useful comments. This material is based upon work supported financially by the National Research Foundation (NRF). Any opinions, findings and conclusions or recommendations expressed in this material are those of the author(s) and therefore the NRF does not accept any liability in regard thereto.

\section{References}

Bailey, J. 1979, MNRAS, 189, 41P

Bloemen, S., Steeghs, D., De Smedt, K., et al. 2013, MNRAS, 429, 3433

Copperwheat, C. M., Marsh, T. R., Dhillon, V. S., et al. 2010, MNRAS, 402, 1824

Harlaftis, E. T., Steeghs, D., Horne, K., Martín, E., \& Magazzú, A. 1999, MNRAS, 306, 348

Harlaftis, E. T., Baptista, R., Morales-Rueda, L., Marsh, T. R., \& Steeghs, D. 2004, A\&A, 417, 1063

Kotze, E. J., \& Potter, S. B. 2015, Acta Polytechnica CTU Proceedings, 2, 170

Marsh, T. R. 2001, in Astrotomography, Indirect Imaging Methods in Observational Astronomy, eds. H. M. J. Boffin, D. Steeghs, \& J. Cuypers (Berlin: Springer Verlag), Lect. Notes Phys., 573, 1

Marsh, T. R., \& Horne, K. 1988, MNRAS, 235, 269

Marsh, T. R., Horne, K., \& Shipman, H. L. 1987, MNRAS, 225, 551

Marsh, T. R., Horne, K., Schlegel, E. M., Honeycutt, R. K., \& Kaitchuck, R. H. 1990, ApJ, 364, 637

Marsh, T. R., Robinson, E. L., \& Wood, J. H. 1994, MNRAS, 266, 137

Osaki, Y. 1974, PASJ, 26, 429

Patterson, J., Richman, H., Kemp, J., \& Mukai, K. 1998, PASP, 110, 403

Savoury, C. D. J., Littlefair, S. P., Marsh, T. R., et al. 2012, MNRAS, 422, 469

Schwope, A. D., Mantel, K.-H., \& Horne, K. 1997, A\&A, 319, 894

Spruit, H. C. 1998, arXiv e-prints [arXiv: astro-ph/9806141]

Spruit, H. C., \& Rutten, R. G. M. 1998, MNRAS, 299, 768

Steeghs, D., Horne, K., Marsh, T. R., \& Donati, J. F. 1996, MNRAS, 281, 626

Steeghs, D., Harlaftis, E. T., \& Horne, K. 1997, MNRAS, 290, L28

Steeghs, D., Howell, S. B., Knigge, C., et al. 2007, ApJ, 667, 442

Warner, B. 1995, Cambridge Astrophys. Ser., 28

Wolf, S., Barwig, H., Bobinger, A., Mantel, K.-H., \& Simic, D. 1998, A\&A, 332, 984 